\section{The GPCR-cancer connection}

\section{By Joanne Kotz, Senior Editor}

Researchers from Roche's Genentech Inc. unit have identified a host of somatic mutations and gene copy number alterations across human cancers that represent potential targets for the disease. ${ }^{1}$ Nineteen of the 112 candidate cancer genes identified are G protein-coupled receptors. With many of these receptors already being targeted in other indications, the path may be open to move rapidly on genes that are validated as cancer targets.

Although previous systematic efforts to sequence somatic mutations in cancer have resulted in new insights into the disease and the identification of new drug targets, these earlier efforts are believed to have only scratched the surface of the cancer genome. ${ }^{2}$

To expand the list of known somatic mutations, a team led by Somasekar Seshagiri, a senior scientist in molecular biology at Genentech, now has sequenced 1,507 cancer-associated or druggable genes, including $156 \mathrm{G}$ protein-coupled receptors (GPCRs), across 441 human primary tumor samples. These were predominantly breast, lung, ovarian and prostate cancers and subtypes.

A total of 2,567 somatic mutations were identified. Protein-altering mutations occurred in 845 genes, $43 \%$ of which had not been previously reported to be mutated in cancer.

"We believe that cataloging changes in tumor genomes is an essential first step in understanding cancer and developing individualized therapeutics to treat it," Seshagiri told SciBX.

Results were published in Nature.

Lynda Chin, a principal investigator for The Cancer Genome Atlas project at the NIH, believes these results complement other cancer genome efforts and confirm "the value of looking broadly and systematically to discover unanticipated genes and insights."

Chin is professor of dermatology at Harvard Medical School and scientific director of the Belfer Institute for Applied Cancer Science and co-leader of the melanoma disease program at the Dana-Farber Cancer Institute.

Although the tumor cohort investigated in the Genentech study is large, Chin cautioned that the small number of samples for each tumor type or subtype limits the ability to detect low-frequency mutations. In addition, insertions, deletions and rearrangements-which she noted "are emerging as important classes of genomic alterations"are not detected by the sequencing approach employed.
Table 1. Potential GPCR cancer targets. Researchers from Roche's Genentech Inc. unit identified 19 G protein-coupled receptors (GPCRs) as potential drug targets for cancer, of which only one appears to be targeted by a compound in the clinic to treat cancer. Zeria Pharmaceutical Co. Ltd. (Tokyo:4559) has Z-360, an oral cholecystokinin B receptor (CCKBR; CCK2R) antagonist, in Phase II for pancreatic cancer.

\begin{tabular}{|c|}
\hline Target \\
\hline Apelin receptor (APLNR; APJ) \\
\hline Brain-specific angiogenesis inhibitor 3 (BAI3) \\
\hline CC chemokine receptor 3 (CCR3; CD193) \\
\hline CCR5 (CD195) - 2 - n \\
\hline Chemokine-like receptor 1 (CMKLR1; ChemR23) \\
\hline Cholecystokinin A receptor (CCKAR; CCK1R) \\
\hline CCKBR \\
\hline G protein-coupled receptor 84 (GPR84) \\
\hline Latrophilin 3 (LPHN3) \\
\hline MAS1 oncogene-like (MAS1L; MRG) \\
\hline Melanin-concentrating hormone receptor 2 (MCHR2; GPR145) \\
\hline Metabotropic glutamate receptor subtype 1 (mGluR1; GRM1) \\
\hline mGluR3 (GRM3) \\
\hline mGluR8 (GRM8) \\
\hline Melanocortin 3 receptor (MC3R) \\
\hline Neuropeptide Y receptor Y2 (NPY2R) \\
\hline NPY5R \\
\hline Prostaglandin F receptor (PTGFR) \\
\hline
\end{tabular}

Purinergic receptor P2Y G protein-coupled 8 (P2RY8; P2Y8)

\section{GPCRs undergo alterations}

Statistical analysis of the genomic data identified 77 proteins with a high frequency of protein-altering mutations. Integrating these mutational data with gene copy number alterations resulted in the identification of 35 additional significantly altered genes. This list of candidate cancer genes includes 19 GPCRs (see Table 1, "Potential GPCR cancer targets").

"While some of the genes in this list were known, there are many genes, including members of the GPCR family, that were not previously known to be altered," Seshagiri told SciBX.

"No prevalent mutations in GPCRs had been previously documented in carcinomas," said J. Silvio Gutkind, chief of the oral pharyngeal cancer branch, cell growth regulation section and molecular carcinogenesis section of the National Institute of Dental and Craniofacial Research of the NIH.

Aberrant overexpression and activation of GPCRs in cancer generally results from increased tumor or stromal cell release of agonists rather than from genomic mutations. "Most prior activating mutations in GPCRs have been reported in the context of endocrine tumors," said Gutkind. He anticipates growing interest in GPCRs in the cancer community.

"The emerging role of gain-of-function mutations in $\mathrm{G}_{\alpha}$ subunits, which are involved in intracellular GPCR signaling, and the present findings of likely oncogenic mutations in GPCRs in highly prevalent 
human malignancies, will surely broaden interest in examining this large family of cell surface receptors as potential targets for cancer treatment," he said.

Fiona Marshall, founder and CSO of Heptares Therapeutics Ltd., echoed this thinking.

"Although GPCRs are well-known drug targets in many disease areas, their role in cancer has been somewhat overlooked whilst many cancer biologists focus on intracellular signaling pathways," she said. "In fact, GPCRs are upstream of many signaling pathways known to be involved in cancer, including the mitogen-activated protein kinase (MAPK), protein kinase B (PKB; Akt) and Ras-Raf pathways. This paper provides further evidence of their involvement in cancer and suggests that some GPCRs may actually be oncogenes."

Heptares is developing mAbs and small molecules that target GPCRs for a range of indications including cancer.

According to Marshall, "all of the GPCRs identified in this study represent potentially druggable targets, either by small molecule or antibody approaches."

\section{Mutation validation}

Establishing a causal connection between mutation and oncogenesis will be the next important step.

The trick, according to Chin, is in prioritizing which genes to investigate first.

"Functional genomics," she said, "can help rank these genes in order of importance for downstream study based not only on statistical values as reported in the current study but also on functional activities from genetic screens."

Chin added that biology and mechanism also will be important. "Significantly more investigation will be required to understand whether a gene is oncogenic or tumor suppressive in what context in cancer," she said.

Marshall also emphasized the need for functional validation and greater biological insight. Questions to be addressed, she said, include: "Are these mutations oncogenic? Do these mutations result in an increase in the level of expression or activity of the receptors and their downstream signaling pathways?"

Gutkind has already singled out one set of GPCRs identified in the study-metabotropic glutamate receptor subtype 1 (mGluR1; GRM1), mGluR3 (GRM3) and mGluR8 (GRM8) - that should be prioritized for exploration as potential therapeutic targets "based on the incidence of mutations and copy number gains and the fact that the members of this receptor family all respond to the same ligand."
If any of these targets can be successfully validated through proof-of-concept animal studies, Marshall sees the potential to rapidly move to clinical testing. "Many of the GPCRs identified in this study are already well-known drug targets for other indications," she said, "and therefore small molecule antagonists are available as tools to evaluate the role of these receptors in cancer."

In particular, Marshall noted that there are clinical antagonists available for some receptors, including neuropeptide $\mathrm{Y}$ receptor $\mathrm{Y} 5$ (NPY5R), CC chemokine receptor 3 (CCR3; CD193) and CCR5 (CD195), and research reagents for a number of the GPCRs identified, including the mGluRs, cholecystokinin receptors and prostaglandin receptors.

Shionogi \& Co. Ltd. has two oral NPY5R antagonists, S-234462 and S-2367, in Phase I/II testing for obesity.

Pfizer Inc. markets the CCR5 antagonist Celsentri maraviroc for HIV/AIDS. At least four other companies have CCR5 antagonists or $\mathrm{mAbs}$ in Phase I/II testing for HIV/AIDS or chronic obstructive pulmonary disease (COPD).

Pharmaxis Ltd. has ASM8, an antisense oligonucleotide that downregulates expression of CCR3 and the receptors for IL-3, IL-5 and granulocyte macrophage colony-stimulating factor (CSF2; GM-CSF), in Phase II testing for asthma.

Genentech declined to disclose which specific targets it is interested in pursuing and did not disclose the patenting or licensing status of the work.

Kotz, J. SciBX 3(31); doi:10.1038/scibx.2010.942

Published online Aug. 12, 2010

\section{REFERENCES}

1. Kan, Z. et al. Nature; published online July 28,2010 ; doi:10.1038/nature09208

Contact: Somasekar Seshagiri, Genentech Inc., South San Francisco, Calif.

e-mail: seshagiri.somasekar@gene.com

2. Stratton, M.R. et al. Nature 458, 719-724 (2009)

3. Dorsam, R.T. \& Gutkind, J.S. Nat. Rev. Cancer 7, 79-94 (2007)

COMPANIES AND INSTITUTIONS MENTIONED

Dana-Farber Cancer Institute, Boston, Mass.

Genentech Inc., South San Francisco, Calif.

Harvard Medical School, Boston, Mass.

Heptares Therapeutics Ltd., Welwyn Garden City, U.K.

National Institute of Dental and Craniofacial Research, Bethesda, Md.

National Institutes of Health, Bethesda, Md.

Pfizer Inc. (NYSE:PFE), New York, N.Y.

Pharmaxis Ltd. (ASX:PXS), French Forest, New South Wales, Australia

Roche (SIX:ROG; OTCQX:RHHBY), Basel, Switzerland

Shionogi \& Co. Ltd. (Tokyo:4507; Osaka:4507), Osaka, Japan 